\title{
Efficient Perovskite Solar Cells by Metal Ion
}

\section{Doping}

\section{AUTHOR INFORMATION}

Jacob Tse-Wei Wang, ${ }^{,}$Zhiping Wang, ${ }^{l}$ Sandeep Pathak, ${ }^{1}$ Wei Zhang, ${ }^{, 1}$ Dane W. deQuilettes, ${ }^{2}$

Florencia Wisnivesky, ${ }^{3}$ Jian Huang, ${ }^{l}$ Pabitra Nayak, ${ }^{l}$ Jay Patel,,${ }^{1}$ Hanis Yusof, ${ }^{4}$ Yana Vaynzof,,${ }^{5,6}$

Rui Zhu, ${ }^{7}$ Ivan Ramirez, ${ }^{1}$ Jin Zhang, ${ }^{l}$ Caterina Ducati, ${ }^{3}$ Chris Grovenor,${ }^{4}$ Michael B. Johnston, ${ }^{1}$ David S. Ginger, ${ }^{2}$ Robin J. Nicholas ${ }^{l *} \&$ Henry J. Snaith ${ }^{l *}$

${ }^{1}$ Department of Physics, University of Oxford, Clarendon Laboratory, Parks Road, Oxford OX1 3PU, UK

${ }^{2}$ Department of Chemistry, University of Washington, Box 351700, Seattle, WA 98195-1700, USA

${ }^{3}$ Department of Materials Science and Metallurgy, University of Cambridge, 27 Charles Babbage Road, Cambridge CB3 OFS, United Kingdom.

${ }^{4}$ Department of Materials, University of Oxford, Parks Road, Oxford, OX1 3PH, UK

${ }^{5}$ Kirchhoff Institute for Physics (KIP), Im Neuenheimer Feld 227, 69120 Heidelberg, Germany

${ }^{6}$ Centre for Advanced Materials (CAM), Im Neuenheimer Feld 225, 69120 Heidelberg, Germany

${ }^{7}$ State Key Laboratory for Artificial Microstructure and Mesoscopic Physics, School of Physics, Peking University, Beijing, China, 100871

$\ddagger$ Current address: School of Chemistry, Joseph Banks Laboratories, University of Lincoln, Beevor Street, Lincoln LN6 7DL, UK.

\section{Corresponding Author}

*r.nicholas1@physics.ox.ac.uk; *henry.snaith@physics.ox.ac.uk 
Keywords: perovskite, hybrid photovoltaics, planar heterojunction, defect chemistry, dopant 


\section{BROADER CONTEXT}

Perovskite solar cells, which promise to deliver the highest efficiency, lowest cost nextgeneration PV technology, have been largely advanced over the last few years by improvements in the polycrystalline thin film quality. So far, improvements in film uniformity and smoothness, have mainly been at the expense of crystalline grain-size, and charge recombination losses at defect sites. High luminescence efficiency, which is an indication of better optoelectronic quality, has generally been found in films with polycrystalline grains of many micrometres in scale. This suggests a current compromise between ideal morphology and ideal optoelectronic quality.

For traditional semiconductors and crystalline solids, the influence of impurity ion doping has been studied extensively and can either alter the crystallisation or induced electronic positive or negative type doping. However, in the perovskite community, impurity doping has been largely unexplored. Here, we show that doping the perovskite solution with $\mathrm{Al}^{3+}$, which has a much smaller ionic radius than $\mathrm{Pb}^{2+}$, has profoundly positive influenced on the crystalline and optoelectronic quality of the perovskite absorber layer: We demonstrate a two-fold increase in the photoluminescence quantum efficiency and a significantly reduced electronic disorder, despite the films still having polycrystalline grains on the order of one micrometer is scale. This largely overcomes the trade-off between film smoothness and optoelectronic quality, and these improvements translate into highly efficient planar heterojunction perovskite solar cells.

Our work paves the way for further improvement of the optoelectronic quality of perovskite thin films, and subsequent devices, via highlighting a new avenue for investigation of the role of dopant impurities upon crystallisation and controlling the electronic defect density in the perovskite thin films. 
ABSTRACT

Realizing the theoretical limiting power conversion efficiency (PCE) in perovskite solar cells requires a better understanding and control over the fundamental loss processes occurring in the bulk of the perovskite layer and at the internal semiconductor interfaces in devices. One of the main challenges is to eliminate the presence of charge recombination centres throughout the film which have been observed to be most densely located at regions near the grain boundaries. Here, we introduce aluminium acetylacetonate to the perovskite precursor solution, which improves the crystal quality by reducing the microstrain in the polycrystalline film. At the same time, we achieve a reduction in the non-radiative recombination rate, a remarkable improvement in the photoluminescence quantum efficiency (PLQE) and a reduction in the electronic disorder deduced from an Urbach energy of only $12.6 \mathrm{meV}$ in complete devices. As a result, we demonstrate a power-conversion efficiency (PCE) of $19.1 \%$ with negligible hysteresis in planar heterojunction solar cells comprising all organic $\mathrm{p}$ and n-type charge collection layers. Our work shows that an additional level of control of perovskite thin film quality is possible via impurity cation doping, and further demonstrates the continuing importance of improving the electronic quality of the perovskite absorber and the nature of the heterojunctions to further improve the solar cell performance.

\section{INTRODUCTION}

Driven by improvements in the quality of organic-inorganic lead halide perovskites (i.e. $\mathrm{CH}_{3} \mathrm{NH}_{3} \mathrm{PbI}_{3}$ ) thin films, perovskite solar cells have achieved a series of efficiency breakthroughs since $2009 .^{1-4}$ Starting from structures based on small nanocrystals embellishing the surface of a 
mesoporous $\mathrm{TiO}_{2}$ scaffold in 2009 , the published highest power conversion efficiency of $21.1 \%$ has been achieved in a device architecture which comprises both a thin layer of mesoporous $\mathrm{TiO}_{2}$ and a thick solid perovskite absorber layer with micrometre size crystalline domains and a high level of uniformity. ${ }^{5}$ In addition, there has been much work on the simplified "planar heterojunction" architecture, where a solid perovskite absorber layer is sandwiched between negative (n) and positive (p) type charge collection layers. ${ }^{2,6}$ One commonality of all this work, is that enhanced crystalline quality and the macroscopic uniformity of the solid perovskite thin films are key parameters which have led to improved device performance. However, there is still much confusion over many factors such as the role and impact of boundaries between the polycrystalline domains, the requirement or not to move towards single crystalline materials, the nature and role of electronic defects in the perovskite absorbers, and the impact of the charge collection layers upon loss processes in the perovskite solar cells.

One specific area which has had some attention is the precise nature of electronic defects in the perovskite films. Yan et al. have used theoretical calculations to propose that many of the defects, such as I or $\mathrm{Pb}$ vacancies, will result in states very close to or within the continuum of states in the conduction and valance bands; therefore only having a minor negative electronic impact upon the solar cells. ${ }^{7}$ However, Agiorgousis et al. have revealed that there is strong covalency between the $\mathrm{Pb}$ cations and I anions in the perovskite plane, which may result in the existence of intrinsic defects, such as undercoordinated $\mathrm{Pb}$ dimers and I trimers, which could generate sites within the band gap and serve as charge recombination centres during solar cell operation. ${ }^{8}$

Previously, post treatment of the perovskite films with Lewis bases, such as pyridine, has been shown to enhance the charge carrier lifetime, ${ }^{9}$ suggesting that pyridine can passivate the 
surface recombination sites, ${ }^{9}$ which are possibly under-coordinated $\mathrm{Pb}$ sites. ${ }^{10}$ However, these sorts of post growth treatments are challenging to control in a reproducible manner, and it is not clear if they are genuinely passivating the defect sites, or simply enabling the perovskite crystal surface to reconstruct in a more favorable manner with fewer crystal surface defects. ${ }^{9}$ On the other hand, incorporation of an impurity "dopant" into the precursor solution itself could provide a more robust way to produce a perovskite film with traps passivated throughout the bulk, or indeed influence the crystallization process in such a way as to minimize the number of defects which result in electronic traps in the first place. ${ }^{11}$ Recently, Sargent and coworkers have shown that addition of Phenyl- $\mathrm{C}_{61}$-butyric acid methyl ester (PCBM) directly into the perovskite precursor solution allows PCBM to sit at the grain boundaries of the processed perovskite films, leading to a certain degree of passivation of the trap states at the grain boundaries, and suppression of hysteresis in the n-i-p planar heterojunction device architecture. ${ }^{12}$ In addition, PCBM is a very good electron acceptor for use as the n-type charge collection material in perovskite solar cells, hence its role may be two-fold when mixed into the perovskite solution. ${ }^{13}$ However, a major challenge to achieving reproducible device performance with this route is the poor solubility and significant phase segregation of PCBM within the perovskite precursor solution. ${ }^{12}$

Here, we investigate the impact of "doping" the perovskite, $\mathrm{CH}_{3} \mathrm{NH}_{3} \mathrm{PbI}_{3}\left(\mathrm{MAPbI}_{3}\right)$, with $\mathrm{Al}^{3+}$, a higher valence metal ion than $\mathrm{Pb}^{2+}$. We introduced a Lewis acid-base coordination complex, aluminium acetylacetonate $\left(\mathrm{Al}-\mathrm{acac}_{3}\right)$, directly into the perovskite precursor solution, which contains $\mathrm{Pb}$ acetate trihydrate $\left(\mathrm{PbAc}_{2} \cdot\left(3 \mathrm{H}_{2} \mathrm{O}\right)\right)$ and methyl ammonium iodide $(\mathrm{MAI}) \cdot{ }^{14} \mathrm{Al}^{3+}$ ions have a much smaller ionic radii than $\mathrm{Pb}^{2+}(53.5 \mathrm{pm}$ vs $119 \mathrm{pm})$ hence we do not expect to observe substitutional doping within the crystal. ${ }^{15}$ However, by careful optimization of the $\mathrm{Al}^{3+}$ 
ion concentration in the perovskite precursor solution, we dramatically enhance the PLQE of the crystalized perovskite films, which increases to $35 \%$ from only $15 \%$ for the non-doped (control) films when measured at $\sim 10$ suns irradiance, which we infer to be due to a reduction in the density of electronic defects. By incorporating these "Al ${ }^{3+}$-doped" films into "inverted" $\mathrm{p}-\mathrm{i}-\mathrm{n}$ perovskite solar cells, we achieve a measured current-density voltage PCE of $19.1 \%$ and a stabilized power output of $18.2 \%$.

\section{RESULTS AND DISCUSSION}

To deposit $\mathrm{Al}^{3+}$-doped $\mathrm{MAPbI}_{3}$ perovskite films, we first prepared a $\mathrm{MAPbI}_{3}$ precursor solution by mixing MAI and $\mathrm{PbAc}_{2} \cdot\left(3 \mathrm{H}_{2} \mathrm{O}\right)$ at a 3:1 molar ratio in dimethylformamide (DMF), with the addition of hypophosphorous acid, as previously reported by Zhang et al. ${ }^{11}$ In order to study the impact of doping concentration on the perovskite, we added varying concentrations (from 0.075 to $1.5 \mathrm{~mol} \%$ of the $\mathrm{Al}^{3+} / \mathrm{Pb}^{2+}$ ratio) of $\mathrm{Al}-\mathrm{acac}_{3}$ into the as prepared $\mathrm{MAPbI}_{3}$ precursor solutions and fabricated a series of perovskite films. We note that, in this manuscript we will refer to the $\mathrm{Al}-\mathrm{acac}_{3}$ addition as " $\mathrm{Al}^{3+}$ doping". We will show evidence towards the end of the manuscript that it is indeed the addition of aluminum ions, and not the organic counter ions which is important for the improvements which we will present. We first evaluate if there is any impact of $\mathrm{Al}^{3+}$ doping upon the crystallization process and the final crystal structure of the resulting perovskite films. In fig. 1a and fig. S1 we show the X-ray diffraction (XRD) spectra for a series of films made with a range of $\mathrm{Al}^{3+}$ doping concentrations up to $1.5 \mathrm{~mol} \%$. All the perovskite films crystallise in a typical tetragonal crystal $(I 4 / \mathrm{mcm}, \mathrm{NO} .140)$ structure as reported previously. ${ }^{1}$ As the doping levels increase up to $0.15 \mathrm{~mol} \%$, the intensities of the dominant perovskite (110) and (220) peaks substantially increase with increasing doping concentration, 
and there is a significant decrease in peak widths (see fig. S2) but no measurable shift in the position of the (110) and (220) diffraction peaks. These results suggest that the $(h h 0)$ planes become increasingly aligned in the presence of the $\mathrm{Al}^{3+}$ dopant. As the doping level rises to 0.3 mol\% concentration, the (110) peak intensity continues to increase, but the peak begins to broaden and shift in position with respect to the control sample. However, upon further increase in doping concentration, the peak intensity begins to decrease and the peak widths continue to broaden. 

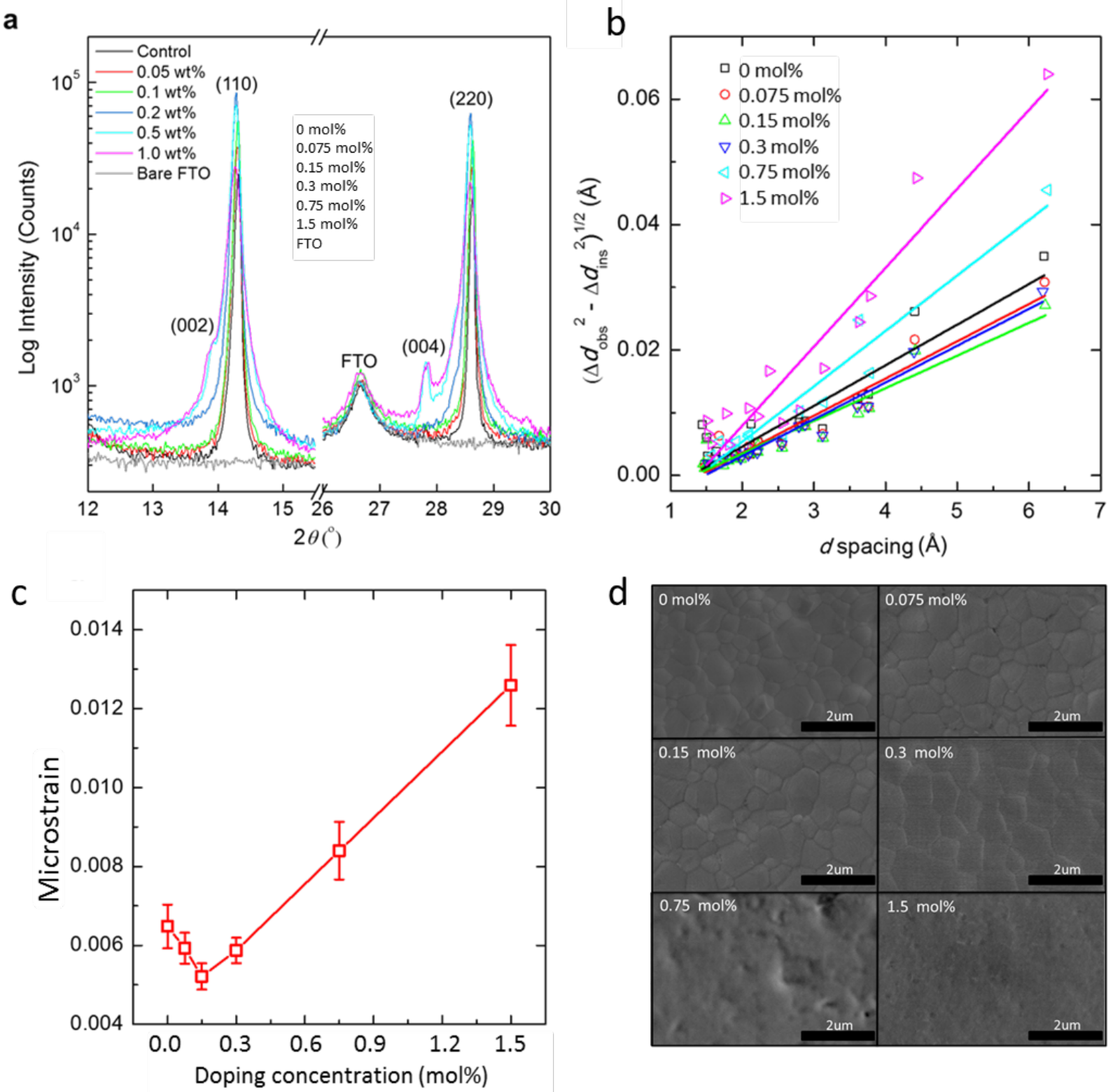

Figure 1. Characterizations of control and $\mathrm{Al}^{3+}$-doped perovskite samples with (a) $\mathrm{X}$-ray diffraction patterns analysis, and (b) A modified Williamson-Hall plot of $\left(\Delta d_{\text {obs }}{ }^{2}-\Delta d_{\text {ins }}{ }^{2}\right)^{1 / 2}$ versus $d$ spacing extracted from the corresponding diffraction profiles shown in Fig. S1. The slopes of the solid lines from linear fits indicate the extents of microstrain at different doping concentrations. (c) Calculated microstrain of samples with different $\mathrm{Al}^{3+}$ doping concentration 
from XRD patterns (The error bars for each concentration in the plot $\mathrm{c}$, are determined from the fits in b). (d) top view scanning electron microscope (SEM).

Broadening and shifts in the XRD peak can be caused by either a reduction in the grain size (Scherrer broadening) and/or non-uniform strain (microstrain). We note that Scherrer broadening will only be significant when the grains are in the range of or less than $100 \mathrm{~nm}$, and as we will discuss later we do not expect this to be a significant contribution here. Strain is the relative change in size of an object with respect to its ideal size (or size before experiencing an external force). The microstrain in a crystalline material is a result of small fluctuations in the lattice spacing, induced by crystal imperfections/structural defects including dislocations, vacancies, stacking faults, interstitials, twinning, and grain boundaries. ${ }^{16,17,18}$ By simply considering Braggs law for scattering of light of wavelength $\lambda, \mathrm{n} \lambda=2 \mathrm{~d} \sin \theta$, it is clear that small fluctuations in d (i.e. $\Delta d$ ) will result in small fluctuations, or broadening, in $\theta$ when measuring the X-ray diffraction from the material. We quantify the extent of microstrain in our perovskite films by analysing the peak broadening in the diffraction patterns according to the modified Williamson-Hall method. ${ }^{17,19}$ The effective observed d-space broadening $\left(\Delta d_{\mathrm{obs}}\right)$ determined from the XRD peak width broadening, is a convoluted function of the Gaussian full width half maximum broadening in the $2 \theta$ scan due to the instrument response $\left(\Delta d_{\text {ins }}\right)$, the grain size $\left(\Delta d_{\text {size }}\right)$ and the microstrain $\left(\Delta d_{\varepsilon}\right)$. These can be de-convoluted from the observed broadening, via,

$$
\Delta d^{2}{ }_{o b s}=\Delta d^{2}{ }_{\varepsilon}+\Delta d^{2}{ }_{\text {ins }}+\Delta d^{2}{ }_{\text {size }}
$$

where the unit-less microstrain $\varepsilon$ is defined as $\varepsilon=\left(\Delta d_{\varepsilon} / d\right)$, where $\mathrm{d}$ is the mean $\mathrm{d}$-spacing. 
Since the perovskite thin films have grains on the order of many hundreds of $\mathrm{nm}$ to microns, it is likely that the size effect induced peak width broadening can be neglected, hence if $\Delta d_{\text {size }}^{2} \ll<\Delta d_{\text {obs }}^{2}{ }^{19}$ and we can write,

$$
\left(\Delta d^{2}{ }_{o b s}-\Delta d^{2}{ }_{i n s}\right)^{1 / 2} \approx \varepsilon d .
$$

Therefore, the slope of $\left(\Delta d^{2}{ }_{o b s}-\Delta d^{2}{ }_{\text {ins }}\right)^{1 / 2}$ versus $d$, gives the magnitude of the microstrain, $\varepsilon$, in the film.

Here we observe in figure $1 \mathrm{~b}$ and $\mathrm{c}$ that introduction of small amounts of $\mathrm{Al}^{3+}$ dopant $(<$ $0.3 \mathrm{~mol} \%$ ) decreases the microstrain. A minimum microstrain of $5.2 \times 10^{-3}$ is achieved at 0.15 mol\%, which is $30 \%$ lower than the control film. We note that this is equivalent to d-spacing fluctuations of the order of $0.5 \%$. This suggests that the introduction of a small amount of $\mathrm{Al}^{3+}$ dopant reduces the density of crystal defects. Additionally, the (110) XRD peak significantly increases in intensity, consistent with the presence of $\mathrm{Al}^{3+}$ enhancing the orientation in the growth of perovskite domains along the (110) plane. ${ }^{20}$

If the $\mathrm{Al}^{3+}$ ions partially substitute $\mathrm{Pb}^{2+}$ ion sites, we would expect the perovskite lattice to be severely distorted due to the much smaller $\mathrm{Al}^{3+}$ ionic radii as compared to the $\mathrm{Pb}^{2+}$ ion, which would induce a shift in $\mathrm{d}^{21}$ Following this reasoning, the unvarying $(h h 0)$ peak position and decreased microstrain imply at the doping level of $0.15 \%$, the $\mathrm{Al}^{3+}$ dopants are unlikely to be incorporated within the perovskite crystal lattice. When the doping concentration increases to 0.3 mol\%, we observe an increase in microstrain, which continues to increase monotonically with the increasing doping concentration.

In fig. 1d we show top-view SEM images of the perovskite samples with different doping concentrations of $\mathrm{Al}^{3+}$. We do not observe any noticeable differences in morphology or apparent grain size in the perovskite films up to doping levels of $0.3 \mathrm{~mol} \%$, compared to the control 
sample. This is consistent with our postulation that the narrowing of the (hh0) diffraction peaks with the doping concentration from zero to $0.15 \mathrm{~mol} \%$ is not due to crystal size effects. Morphologically, it appears that the perovskite films can tolerate the addition of $\mathrm{Al}^{3+}$ ions to the precursor solution up to around $0.3 \mathrm{~mol} \%$, before any negative impact upon the morphology occurs. The disappearance of the polycrystalline grains in the SEM images, with $\mathrm{Al}^{3+}$ concentrations at or above $0.75 \mathrm{~mol} \%$, is consistent with these films having much smaller polycrystalline domains, which are not clearly discernible in the SEM image. We also note that the XRD patterns for the films with over 0.3 mol\% doping, which we have shown in Fig. 1a, are strongly perturbed, indicating a limit to the concentration of $\mathrm{Al}^{3+}$ which the perovskite films can tolerate.

To probe whether $\mathrm{Al}$ ions remain in the annealed films, we performed inductively coupled plasma optical emission spectrometry (ICP-OES). The detection limits of ICP-OES typically range from parts per million (ppm) to parts per billion (ppb) ${ }^{22}$ For the sample with 0.15 mol\% $\mathrm{Al}^{3+}$ doping (the calculated $\mathrm{Al} / \mathrm{Pb}$ is $2.6: 1000$ ), we determine a molar ratio of $\mathrm{Al} / \mathrm{Pb}$ of 1.2:1000 from the ICP-OES analysis, which confirms the presence of $\mathrm{Al}^{3+}$ in the doped film, only at a slightly lower concentration than in the starting solution. On the contrary, for the control film we determine an $\mathrm{Al} / \mathrm{Pb}$ molar ratio of 0.016:1000, which is almost negligible, as expected. We further validated this finding for higher concentration solutions $(>0.3 \mathrm{~mol} \%)$ using X-ray photoelectron spectroscopy (see fig. S3).

To further investigate the location and distribution of the $\mathrm{Al}^{3+}$ ions within the perovskite film, we employed nanoscale secondary ion mass spectrometry (NanoSIMS) to perform elemental mapping for the doping level of $0.15 \mathrm{~mol} \%$. The NanoSIMS system uses a $16 \mathrm{KeV}$ 
primary ${ }^{133} \mathrm{Cs}^{+}$beam to bombard the perovskite film surface, and then collects and analyzes the Al signal as a negative ion which is commonly detected as $\mathrm{AlO}^{-}$(the oxygen is captured from the sample surface or the vacuum system). In fig. S4 we show the secondary electron SEM images overlaid with the elemental map for Al. In the secondary electron image the darker regions are regions of thicker films, which we therefore interpret as being the centre of grains, and the lighter regions are the thinner regions which we therefore interpret to be the grain boundaries. Although the Al signal is apparent everywhere in the film, it is most strongly concentrated near the brighter regions of the secondary electron microscopy image, indicative of increased Al concentration near the grain boundaries. This is consistent with the $\mathrm{Al}^{3+}$ ions being predominantly expelled to the perovskite surface and grain boundaries during crystallisation.

Considering all the evidence we have presented above, it appears that the presence of a small amount of $\mathrm{Al}^{3+}$ in the perovskite growth solution results in better crystallisation with resultantly fewer crystal defects, as we infer from the reduced microstrain. However, beyond an optimum level of addition of $\mathrm{Al}^{3+}$, the crystallization is strongly perturbed resulting in increased levels of defects. At low $\mathrm{Al}^{3+}$ doping concentrations, the $\mathrm{Al}^{3+}$ ions appear not to be incorporated into the perovskite lattice, but predominantly expelled to the surface and grain boundaries. The precise mechanism by which the crystallisation is enhanced with a small quantity of $\mathrm{Al}^{3+}$ doping is difficult to determine. However, based on our investigation above, we describe our postulated mechanism here: The presence of impurity ions is known to influence crystallisation of materials since the earliest investigations of crystal growth. ${ }^{23}$ Impurity ions can either slow down crystallisation by adsorbing to crystal surfaces and sterically inhibiting growth, or the impurity ions can accelerate crystal growth by changing the surface energy of the crystal growth front making it more favourable for accelerated crystallization. ${ }^{24-26}$ In the low concentration regime 
(below $0.3 \mathrm{~mol} \% \mathrm{Al}^{3+}$ ) we infer that the $\mathrm{Al}^{3+}$ is facilitating enhanced crystallization, and specifically the growth of $(h h 0)$ crystal planes, resulting in more orientated crystalline domains. In fig. 2, we show a schematic diagram of the proposed perovskite growth mode and influence of the $\mathrm{Al}^{3+}$ doping. We also illustrate crystal defects which could potentially exist at the edges of the perovskite crystal surface in the form of terraces, and indicate how the $\mathrm{Al}^{3+}$ doping can reduce the prevalence via the selective enhancement of growth of these terraces.

In order to visualize how the $\mathrm{Al}^{3+}$ doping has influenced the as-crystalized films, we performed atomic force microscopy (AFM) measurements on our samples. We show these images in Figure 2, and plot histograms for the distribution in heights from the images. The lower regions correspond to the troughs at the boundary of the grains, and the higher regions correspond to the top most surfaces of the grains. For the control sample we observe a symmetric, approximately Gaussian distribution of heights, indicating that the surface of the perovskite film is continuously undulating over the measured length-scale, with no specific preferred height. We estimate a half-width at half-maximum (HWHM) height distribution of 33 $\mathrm{nm}$. This level of undulation is almost identical to the underlying FTO substrate, which we determine a HWHM height distribution of $34 \mathrm{~nm}$, which we show in the SI. Thus the control perovskite film does not appear to planarize the FTO. In contrast for the optimum $(0.15 \mathrm{~mol} \%)$ doped perovskite sample, the height distribution becomes asymmetric with a background undulation of the same extent, but with an additional distribution with a much narrower HWHM of $\sim 16 \mathrm{~nm}$ on the top-most region of the films. This indicates that the top of the grains are much flatter in the $\mathrm{Al}^{3+}$ doped samples than in the control samples. The height distribution for the $1.5 \%$ $\mathrm{Al}^{3+}$ doped sample significantly changes, but still shows a relatively narrow distribution on the top surface and a long tail indicating a relatively flat film, but now with the presence of pinholes. 
Since the crystal morphology of the $1.5 \%$ sample has changed considerably, we can-not compare this directly to the other two compositions. These observations further corroborate that, with low levels of $\mathrm{Al}^{3+}$ doping, the perovskite crystalline growth is indeed enhanced and preferentially selects certain planes, while in addition the reduced roughness of the surface of the grains is consistent with a reduction in terrace/step-like defects on the crystal surface.

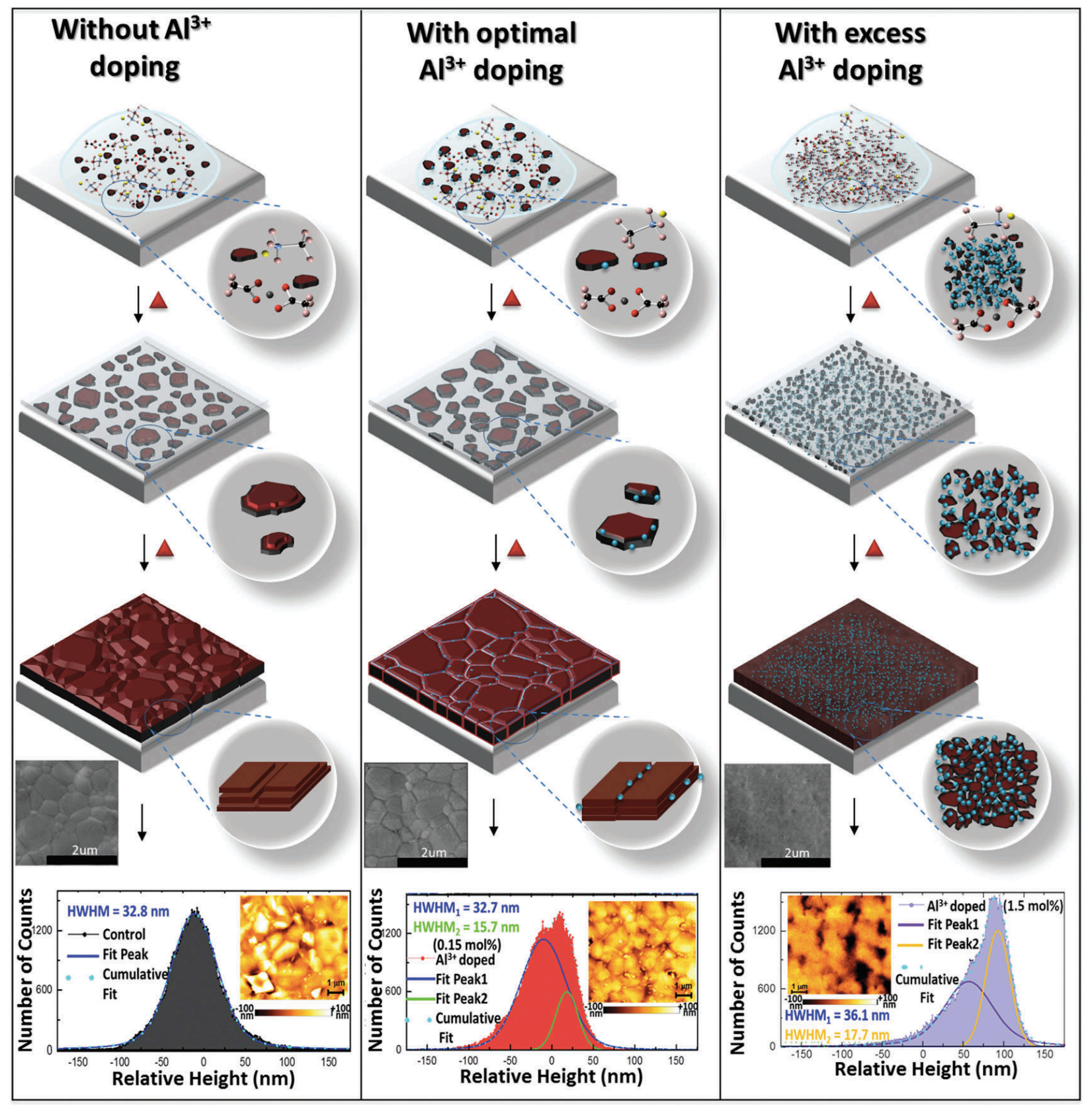


Figure 2: Schematic diagram of the proposed perovskite polycrystalline thin film growth and influence of the $\mathrm{Al}^{3+}$ doping with illustrated $\mathrm{C}, \mathrm{N}, \mathrm{H}, \mathrm{O}, \mathrm{I}, \mathrm{Pb}$, and $\mathrm{Al}$ atoms are indicated by the color of black, blue, pink, red, grey, and cyan respectively; And histograms of the surface topography and the images (insets) from AFM measurements of different samples. Best fits for the histograms are obtained using one and two Voigt distributions, respectively for the control and the optimized sample. The individual distribution components with their HWHM, and the cumulative fits are labelled as in the figure.

We now investigate if the structural improvements in the $\mathrm{Al}^{3+}$-doped polycrystalline films translate to enhanced optoelectronic properties. To understand the influence of $\mathrm{Al}^{3+}$ doping upon the photo-physical properties of the perovskite films we performed absorption and photoluminescence measurements, which we show in fig. S5. We observe little change in the absorption spectra for the films, but we do observe an increase in steady-state photoluminescence (PL) intensity with a maximum for the $0.15 \mathrm{~mol} \% \mathrm{Al}^{3+}$-doped samples, and a subsequent fall off of in intensity upon further increase in doping concentration. In fig. 3 we compare the PL properties of the control films and those doped with $0.15 \mathrm{~mol} \% \mathrm{Al}^{3+}$. In fig. $3 \mathrm{a}$ and $3 \mathrm{~b}$ we observe that the $\mathrm{Al}^{3+}$-doped perovskite films have higher steady state PL intensity and an almost two-fold increase in PL life time compared to the control. 

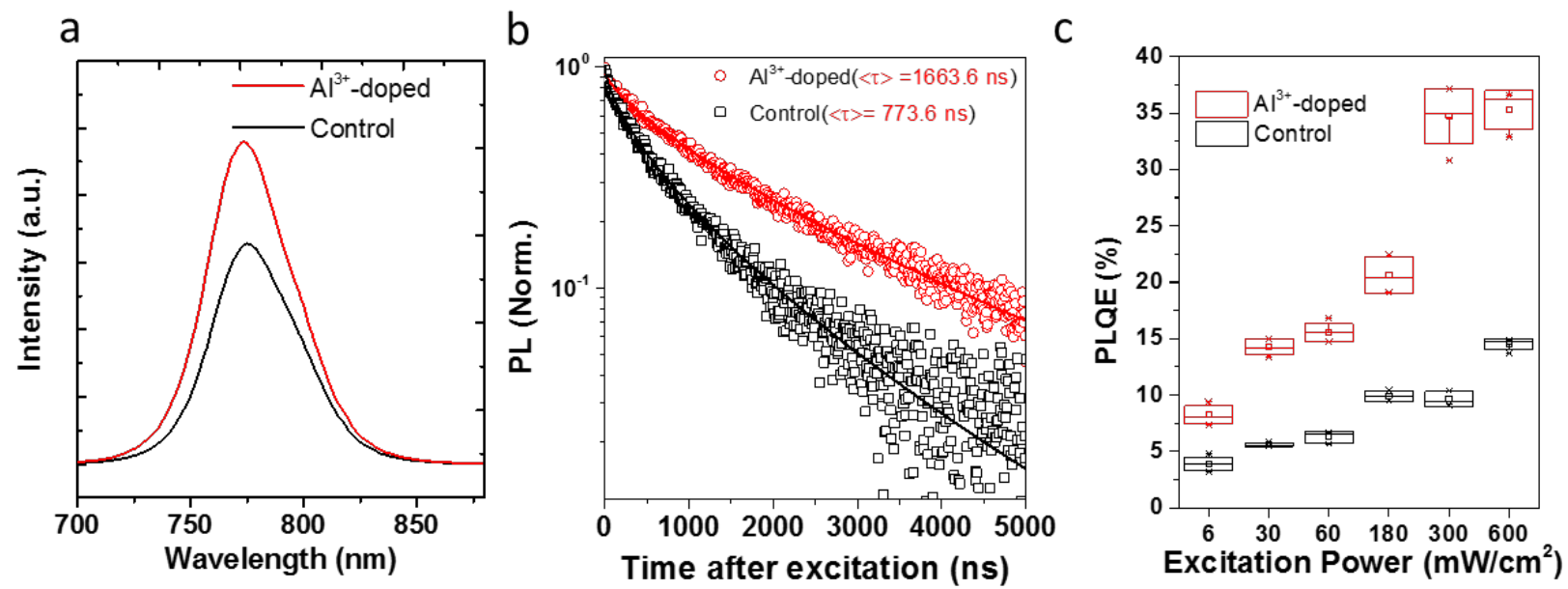

Figure 3: Photoluminescence spectroscopy of control (non-doped) and $0.15 \mathrm{~mol} \% \mathrm{Al}^{3+}$-doped perovskite thin films deposited on glass substrate with a) steady-state spectra $\left(300 \mathrm{~mW} / \mathrm{cm}^{2}, 507\right.$ $\mathrm{nm})$, b) time-resolved photoluminescence decay after excitation at $507 \mathrm{~nm}$ with pulsed (200 $\mathrm{kHz}$ ) and a fluence of $30 \mathrm{~nJ} / \mathrm{cm}^{2} /$ pulse, and c) photoluminescence quantum efficiency (PLQE) as a function of excitation power (further details are available in the supplementary materials).

To further quantify the influence of the $\mathrm{Al}^{3+}$ doping upon the radiative and non-radiative recombination rates, we performed photoluminescence quantum efficiency (PLQE) measurements as a function of excitation power, which we show in fig. 3c. For both doped and control samples, the PLQE rises with increasing excitation power, a behaviour attributed to the filling of trap states and increasingly favourable competition of radiative bimolecular recombination over non-radiative trap assisted recombination. ${ }^{10,27,28}$ Notably, the PLQE is higher for the $\mathrm{Al}^{3+}$ doped samples at all light intensities, which, along with the increase we observe in the PL lifetime, is consistent with fewer fast non-radiative decay channels in the $\mathrm{Al}^{3+}$-doped samples. We fit the bulk time-resolved PL data in fig. $3 \mathrm{~b}$ with a stretched exponential function and extract the average lifetimes, $<\tau>$, as previously described. ${ }^{9}$ We use these low-fluence PL lifetime values along with the low excitation power $\left(6 \mathrm{~mW} / \mathrm{cm}^{2}\right)$ PLQE values, where in both 
cases we expect the carrier recombination to be dominated by pseudo first-order kinetics, ${ }^{9,28}$ to estimate the radiative and non-radiative decay rates in the films using,

$$
P L Q E=\left(\frac{k_{\text {rad }}}{k_{\text {rad }}+k_{\text {non-rad }}}\right),
$$

where $k_{\text {rad }}$ is the radiative decay rate $k_{\text {non-rad }}$ is the non-radiative decay rate and $k_{\text {rad }}+k_{\text {non-rad }}=1 /<\tau>$. For the control and $\mathrm{Al}^{3+}$-doped films, the $k_{\text {rad }}$ we determine are similar for the two films at $5.04 * 10^{4} \mathrm{~s}^{-1}$ and $4.96 * 10^{4} \mathrm{~s}^{-1}$, while we observe a reduction in $\mathrm{k}_{\text {non-rad }}$ from $1.24 * 10^{6} \mathrm{~s}^{-1}$ to $5.52 * 10^{5}$ $\mathrm{s}^{-1}$ for the control and $\mathrm{Al}^{3+}$ doped films. This indicates that the substantive change in the photophysical properties of the perovskite films with $0.15 \mathrm{~mol} \% \mathrm{Al}^{3+}$ is approximately a twofold reduction in the non-radiative recombination rate. We note that both the radiative and nonradiative decay rates are relatively low for a crystalline semiconductor. The slow recombination in metal halide perovskites is a highly fortuitous property for PV devices, but the origin of the phenomenon remains to be elucidated..$^{29}$

The above analysis gives an indication of the macroscopic changes occurring within the perovskite films. However, we know that severe photophysical heterogeneity can occur within the polycrystalline films, and hence to clarify which microscopic regions of the film are responsible for the reduced non-radiative decay, we employ confocal fluorescence microscopy. In fig. $4 \mathrm{a}$ and b we show a $10 \times 10 \mu \mathrm{m}$ confocal fluorescence image of a control and $0.15 \mathrm{~mol} \%$ $\mathrm{Al}^{3+}$-doped perovskite film. We show the image histograms of occurrence versus PL (counts) in fig. 4c and d. In both films there exists heterogeneity in the PL emission with both bright and dark grains, and generally lower levels of PL in the regions near the grain boundaries. From the histograms it is apparent that the average number of PL counts (solid lines) of the $\mathrm{Al}^{3+}$ doped films have shifted to higher levels, but the distribution is similarly broad to that seen in the 
control. In fact, the distribution has slightly broadened indicating no preferential spatial homogenisation of the PL, but simply an overall reduction in the non-radiative decay, both on or in the grains and at the grain boundaries. In Fig. S6, we performed Kelvin probe measurement in the dark and under illumination with perovskite films on FTO, and find a reduction in the surface photovoltage for $\mathrm{Al}^{3+}$-doped films compared to control films, consistent with the removal of electronic traps in the former.

In order to quantify the electronic disorder in the films, we estimate the Urbach energy $\left(E_{u}\right)$ of perovskite films from Fourier transform photocurrent spectroscopy, which we show in fig. S7. We estimate the $\mathrm{E}_{\mathrm{u}}$ for the control and $0.15 \mathrm{~mol} \% \mathrm{Al}^{3+}$-doped samples to be $13.09 \pm 0.05$ and $12.64 \pm 0.04 \mathrm{meV}$ respectively. This decrease in $\mathrm{E}_{\mathrm{u}}$ is direct evidence for an enhancement of the electronic quality of the perovskite films by $\mathrm{Al}^{3+}$ doping. Notably, this is the first time an $\mathrm{E}_{\mathrm{u}}$ has been determined to be less than $13 \mathrm{meV}$ in a metal halide perovskite film, indicating the high electronic quality of the perovskite film ${ }^{11,30}$, and suggesting an improved crystalline order in the polycrystalline thin film. The reason why the steepness of the absorption onset, and ultimately EQE spectra is important, is that this governs the maximum open-circuit voltage a solar cell can achieve in the "radiative limit", where all non-radiative recombination is switched off. We note that the first report of the $\mathrm{E}_{\mathrm{u}}$ in perovskite films estimated a value of $15 \mathrm{meV}$ at room temperature. This is comparable to the $\mathrm{E}_{\mathrm{u}}$ in crystalline silicon, but larger than the room temperature value in GaAs, which is as low as $7 \mathrm{meV}$. However, the significant reduction to $12.6 \mathrm{meV}$ so far, indicates that we have not yet reached the lower limit, and it may be possible that perovskites will end up closer to GaAs than crystalline silicon. 

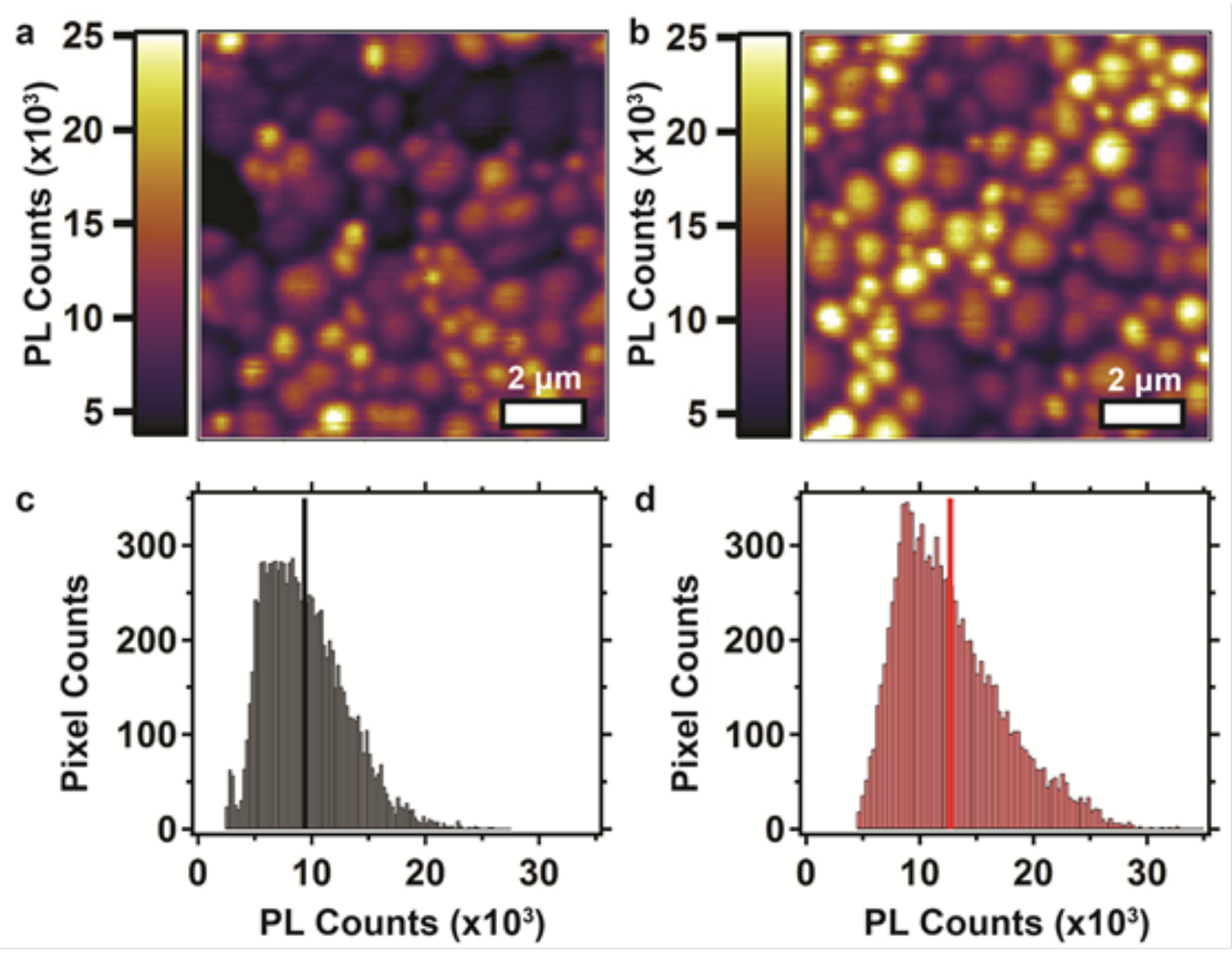

Figure 4: Fluorescence microscopy of (a) control and (b) $\mathrm{Al}^{3+}$-doped (0.15 mol\%) perovskite films with $10 \times 10 \mu \mathrm{m}$ area mapping excited with a pulsed $470 \mathrm{~nm}$ laser (40 $\mathrm{MHz}, 117$ ps pulse width, $300 \mathrm{~nJ} / \mathrm{cm}^{2} /$ pulse $)$. (c) and (d) are the image histograms (10,000 pixels) showing the fluorescence emission distribution with solid lines indicate the average number of PL counts. The $\mathrm{Al}^{3+}$-doped perovskite has more pixel counts falling under "bright" regions as compared to the control. 
By interpreting the photo-physical and electronic impact of the $\mathrm{Al}^{3+}$ doping, it is apparent that the optimally doped films possess fewer non-radiative decay sites and are of superior electronic quality as compared with the control films. This is consistent with the enhanced structural quality we observed in fig. 1, resulting in improved optoelectronic performance. To assess if these enhanced film properties translate into enhancements in solar cell performance, we now integrate the perovskite films into "inverted" (p-i-n) planar heterojunction perovskite solar cells with a p-type organic hole transporting material (HTM), a solid "intrinsic" (i) perovskite film and an n-type organic electron transporting material. ${ }^{31}$ We show a high angle annular dark field STEM (HAADF-STEM) cross-sectional micrograph of an optimized device in fig. 5a, an illustration of the energy levels of the materials employed in fig. $5 \mathrm{~b}$ and a schematic of the full device architecture in fig. 5c. We note that the energy levels we illustrate are for the individual isolated materials, and upon doping and contact with each other there is likely to be a relative shift in the absolute energy at the interfaces. ${ }^{32}$
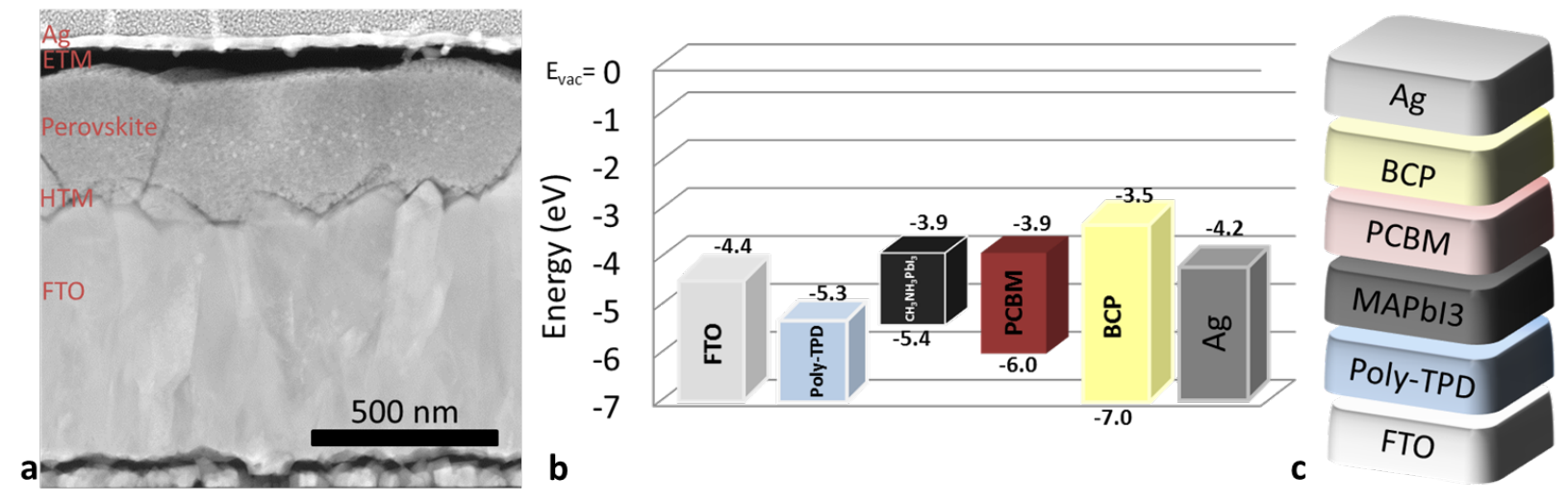

Figure 5: (a) High angle annular dark field STEM (HAADF-STEM) cross-sectional micrograph of optimized device with the structure of $\mathrm{Ag} / \mathrm{BCP} / \mathrm{ETM}$ (PCBM)/perovskite/HTM (polyTPD)/FTO. (b) Diagram of the energy levels of the materials used and (c) an illustration of the structure of the solar cells. 
In fig. S8 and in Table S1 we show the mean solar cell performance parameters for a batch of solar cells with a range of $\mathrm{Al}^{3+}$ doping concentrations, extracted from $\mathrm{JV}$ curves measured under simulated AM 1.5, $100 \mathrm{~mW} / \mathrm{cm}^{2}$ irradiance. We observe a clear trend of enhancement in device performance produced by the introduction of $\mathrm{Al}^{3+}$ dopant in to the perovskite precursor solution up to concentrations of around 0.15 to $0.3 \mathrm{~mol} \%$. For devices fabricated from the control precursor solution, i.e. pristine $\mathrm{MAPbI}_{3}$, the average short-circuits current density $\left(\mathrm{J}_{\mathrm{sc}}\right)$ and open circuit voltage $\left(\mathrm{V}_{\mathrm{oc}}\right)$ are around $19.0 \pm 1.7 \mathrm{~mA} / \mathrm{cm}^{2}$ and $1.01 \pm 0.04 \mathrm{~V}$, respectively. With the incorporation of the $\mathrm{Al}^{3+}$ dopant in the perovskite, both the $\mathrm{J}_{\mathrm{sc}}$ and $\mathrm{V}_{\mathrm{oc}}$ increase up to average values of $21.2 \pm 1.1 \mathrm{~mA} / \mathrm{cm}^{2}$ and $1.06 \pm 0.03 \mathrm{~V}$, respectively, peaking at $0.15 \mathrm{~mol} \%$, while we observe that the average fill factor (FF) increases to $0.72 \pm 0.08$ with the addition of $\mathrm{Al}^{3+}$ up to $0.3 \mathrm{~mol} \%$, after which the FF begins to decrease. Hence, we observe the power conversion efficiency (PCE) to reach a maximum at $0.15 \mathrm{~mol} \%$ of $\mathrm{Al}^{3+}$ doping, with an average PCE of the solar cells fabricated with this doping concentration of $15.8 \pm 2.4 \%$. Notably, the optimized $\mathrm{Al}^{3+}$ doping not only improves all of the performance parameters (i.e. $\mathrm{FF}, \mathrm{V}_{\mathrm{oc}}, \mathrm{J}_{\mathrm{sc}}$ and PCE) as compared with the control devices, but also enhances device reproducibility, as we show in fig. S9.

In fig. 6a we show the current-density voltage (JV) curves for the most efficient control and $\mathrm{Al}^{3+}$ doped devices, where the latter exhibits a $J_{\mathrm{sc}}$ of $22.4 \mathrm{~mA} / \mathrm{cm}^{2}$, a FF of 0.78 , and a $\mathrm{V}_{\text {oc }}$ of $1.10 \mathrm{~V}$ yielding a PCE of $19.1 \%$. When we compare this with the control device, we can see that the major improvement is coming from the increases in $\mathrm{V}_{\mathrm{oc}}$ and $\mathrm{J}_{\mathrm{sc}}$. The external quantum efficiency (EQE) spectra for typical cells of each type, which we show in fig. 6b, exhibit the same trend as the measured JV curves, consistent with the enhanced photocurrent from the $\mathrm{Al}^{3+}$ doped samples. Integrating the EQE over the AM1.5 solar spectrums at $100 \mathrm{mWcm}^{-2}$ irradiance gives an 
estimated $\mathrm{J}_{\mathrm{sc}}$ of 20 and $18 \mathrm{mAcm}^{-2}$ for the $\mathrm{Al}^{3+}$ doped and control devices, respectively. The measured $\mathrm{J}_{\mathrm{sc}}$ under the solar simulator for these precise cells was 22.0 and $17.3 \mathrm{mAcm}^{-2}$ respectively, which gives an indication of an accuracy of between -10 and $+4 \%$, comparing the current estimated from the EQE to the JV measurements under simulated sun light. This difference may be within the measurement accuracy since we use two different rigs with two different calibration diodes. The cells are masked in the same manner for both measurements, however, the spatial uniformity of the EQE measurement is not as controlled as it is for the JV measurements, which may contribute to the errors. Additionally, we note that we test these cells in air without encapsulation, and there is usually a little degradation in performance for these $\mathrm{p}$-i$\mathrm{n}$ inverted cells following testing.

The JV curves for both control and $\mathrm{Al}^{3+}$-doped perovskite solar cells exhibit very low levels of hysteresis. We illustrate this in fig. 6c showing the numerically averaged JV curves measured at different voltage sweep directions in a selection of 20 high performance devices with their performance parameters in the inset table. As an aside, this observation further strengthens our understanding that the main source of such hysteresis originates at the interface between the perovskite film and the semiconducting metal oxide such as $\mathrm{TiO}_{2}$ or $\mathrm{ZnO}$ in regular device structures, probably due to the generation of a large density of electronic defects at the surface of the perovskite when the films are crystallised on these metal oxide surfaces ${ }^{33}$ Here, these cells using only organic $\mathrm{p}$ and $\mathrm{n}$-type charge collection layers exhibit very well behaved current voltage characteristics. Notably, we have recently identified that the presence of electronic defects through which trap assisted recombination occurs, are central to the mechanism governing JV hysteresis in perovskite solar cells (combined with mobile ionic 
species) ${ }^{33}$ Our results are therefore consistent with the notion that perovskite films processed on organic semiconductors contain fewer electronic defects, than those processed on metal oxides.

Table 1. Best device performance parameters with different $\mathrm{Al}^{3+}$ doping concentration under simulated AM 1.5, $100 \mathrm{~mW} / \mathrm{cm}^{2}$ solar irradiation.

\begin{tabular}{|c|c|c|c|c|}
\hline $\begin{array}{c}\mathrm{Al}^{3+} \text { doping } \\
\text { concentration } \\
\text { (mol\%) }\end{array}$ & $\mathrm{J}_{\mathrm{sc}}\left(\mathrm{mA} / \mathrm{cm}^{2}\right)$ & $V_{\text {oc }}(V)$ & FF (\%) & PCE (\%) \\
\hline 0 & -21.3 & -1.04 & 77.2 & 17.1 \\
\hline 0.075 & -21.1 & -1.07 & 78.0 & 17.7 \\
\hline 0.15 & -22.4 & -1.10 & 77.6 & 19.1 \\
\hline 0.3 & -21.8 & -1.07 & 74.9 & 17.5 \\
\hline 0.75 & -20.3 & -1.01 & 65.2 & 13.4 \\
\hline 1.5 & -9.9 & -1.04 & 70.1 & 7.3 \\
\hline
\end{tabular}


In fig. 6d, we show the stabilised power output (SPO) of the best performing control and $\mathrm{Al}^{3+}$ doped cells when holding the applied bias fixed at its maximum power point voltage under simulated AM $1.5\left(100 \mathrm{~mW} / \mathrm{cm}^{2}\right)$ sun light, and measuring the photocurrent as a function of time.

Both devices reach their stabilised photocurrent within the first measurement window, indicative of negligible hysteresis. ${ }^{34}$ For the $\mathrm{Al}^{3+}$-doped device, we measure a stabilized photocurrent at $20.3 \mathrm{~mA} / \mathrm{cm}^{2}$ and a stabilised power output (SPO) of $18.2 \%$, which is superior to the control device for which we measure a stabilized photocurrent and SPO of $18.2 \mathrm{~mA} / \mathrm{cm}^{2}$ and $15.7 \%$, respectively. We note that despite the negligible hysteresis in the JV curves, we still have slightly lower SPOs than the PCE determined from the JV curves. This emphasises that SPO measurements remain important even in cells appearing to exhibit negligible hysteresis. In fig. S10, we further show the average-SPO in a selection of 20 high performance devices at $17.7 \%$ and $15.6 \%$ for the $\mathrm{Al}^{3+}$-doped and control devices respectively. 

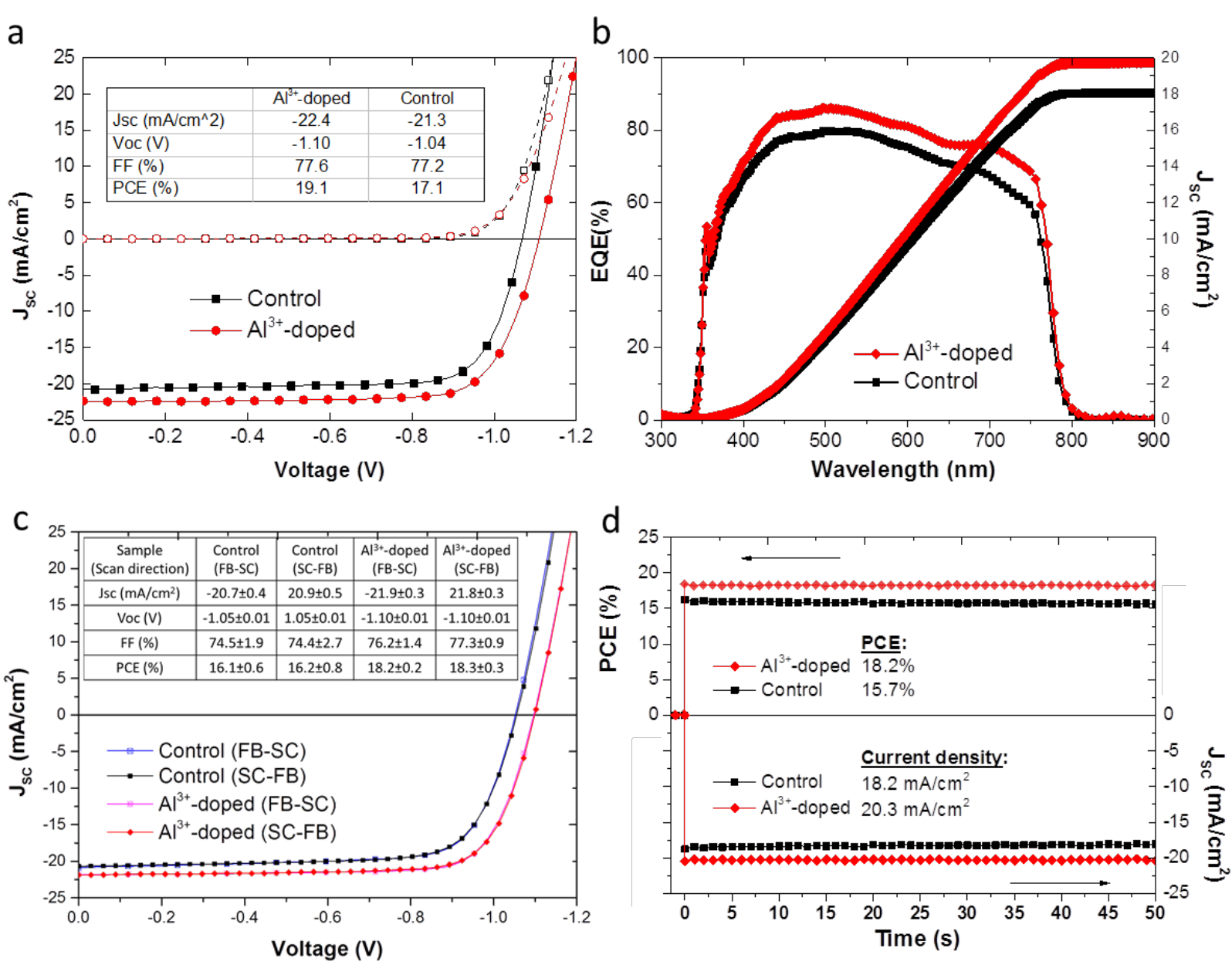

Figure 6: Photovoltaic performance of control and $\mathrm{Al}^{3+}$-doped $(0.15 \mathrm{~mol} \%)$ perovskite solar cells under simulated AM 1.5, $100 \mathrm{~mW} / \mathrm{cm}^{2}$ solar irradiation (solid line), and in the dark (dotted line) at a scan rate of $0.38 \mathrm{Vs}^{-1}$. (a) the current density-voltage characteristics of the best cells. (b) external quantum efficiency (EQE) spectrum measured at short-circuit, along with the expected photocurrent integrated over the AM1.5 solar spectrums of $100 \mathrm{~mW} / \mathrm{cm}^{2}$. (c) Numerically averaged $J V$ curves measured from forward bias (FB) to short-circuit (SC) and back again without observable hysteresis in a selection of 20 high performance control and $\mathrm{Al}^{3+}$ doped devices. (d) Stabilized maximum power output measurement of the best cells with bias at their maximum power points. 
To confirm that the positive effects we observe here are due to the addition of $\mathrm{Al}^{3+}$, as opposed to the acac ion, we also replaced the $\mathrm{Al}(\mathrm{acac})_{3}$ dopant with Aluminum iodide ( $\mathrm{AlI}_{3}$ ). These films exhibit enhanced luminescent properties, and subsequently deliver improved solar cells in comparison to the control devices. We show these results in fig. S11 where we observe that the addition of $\mathrm{AlI}_{3}$ into perovskite precursor solution also delivers optimum performance with a doping level of around $0.15 \mathrm{~mol} \%$.

\section{CONCLUSION}

To summarize our findings, we have discovered that the electronic quality of the $\mathrm{MAPbI}_{3}$ films can be substantially enhanced by low levels of doping with the small metal cation $\mathrm{Al}^{3+}$. We have proposed the mechanism by which $\mathrm{Al}^{3+}$-doping enhances the quality of the films, and revealed a simple property of the material, microstrain, which can be studied, tuned and minimised to deliver even higher quality perovskite films for optoelectronics. With the presence of $\mathrm{Al}^{3+}$ during the perovskite crystallization, we observe reduced microstrain in the perovskite crystal, which we infer to indicate a reduction in the density of crystal defects. From these same films we observe enhanced luminescent properties and reduced electronic disorder, which we infer to suggest that the same defects responsible for microstrain in the crystal, are also responsible for losses in luminescence and the solar cell operation. In addition, the remaining $\mathrm{Al}^{3+}$ ions present, most concentrated in the regions near the grain boundaries, may also act to alter the energy of these defect sites making them more benign. However, this latter consideration is not necessary to explain our results and is only a postulation. We have demonstrated improved JV measured PCE of up to $19.1 \%$ with negligible hysteresis for these $\mathrm{Al}^{3+}$-doped films, when integrated into planar heterojunction solar cells. We attribute the improved operation to the reduced non-radiative trap assisted recombination in the thin films and 
devices. Our work therefore highlights the possibility for considerable further improvement of the electronic quality of perovskite thin films, and subsequent devices by understanding the role of dopant impurities upon crystallisation, and crystal and electronic defects in perovskite thin films.

\section{METHODS}

\section{Perovskite solution preparation}

To form the control methylammonium lead iodide $\left(\mathrm{MAPbI}_{3}\right)$ precursor solution, it was prepared by mixing methylammonium iodide (Dyesol) and lead acetate trihydrate (Aldrich) at a 3:1 molar ratio, then dissolved in anhydrous N,N-Dimethylformamide with final concentrations of $30 \mathrm{wt} \%$ with the addition of hypophosphorous acid $(3 \mathrm{ul} / \mathrm{ml})$ as previously reported by Zhang et al. ${ }^{11}$ For $\mathrm{Al}^{3+}$-doped methylammonium lead iodide solution preparation, Aluminium acetylacetonate $\left(\mathrm{Al}-\mathrm{acac}_{3}\right.$ ) was added into the $\mathrm{MAPbI}$, precursor solution by desired $\mathrm{Al}^{3+} / \mathrm{Pb}^{2+}$ ratios ranging from 0.075 to $1.5 \mathrm{~mol} \%$. (i.e. we add $3 \mathrm{mg}$ of $\mathrm{Al}-\mathrm{acac}_{3}$ into $1 \mathrm{~g} \mathrm{MAPbI}_{3}$ precursor solution to achieve the $1.5 \mathrm{~mol} \% \mathrm{Al}-\mathrm{acac}_{3}$ doping.)

\section{Device fabrication}

All the devices here were fabricated on piranha cleaned fluorine doped tin oxide (FTO) coated glass serving as the transparent electrode. We then deposit the hole-transporter material (HTM) of $\sim 20 \mathrm{~nm}$ thickness by spin-coating a solution of F4-TCNQ-doped poly-TPD (1Material Inc.) following the work of Wang et.al. ${ }^{35}$ Then the perovskite layer was prepared by spin-coating at $2000 \mathrm{rpm}$ for $45 \mathrm{~s}$ from as-prepared precursor solution, drying the substrates for $10 \mathrm{~min}$ on the bench in the glovebox, followed by annealing at $100^{\circ} \mathrm{C}$ for $5 \mathrm{~min}$. For the $\mathrm{n}$-type 
collection layers, PCBM (dissolved in DCB, $2 \mathrm{wt} \%$ ) was spin-coated on top of the perovskite films at $1000 \mathrm{rpm}$ for 30s. Followed by the deposition of a thin $(\sim 5 \mathrm{~nm})$ buffer layer of bathocuproine (BCP, $0.5 \mathrm{mg} / \mathrm{ml}$ in IPA) by spinning at $6000 \mathrm{rpm}$ for 20 seconds. Finally, devices were completed by thermal evaporation of $70 \mathrm{~nm}$ of silver contacts under high vacuum.

\section{ASSOCIATED CONTENT}

\section{Supplementary Information.}

Full Experiment details, microstrain analysis, device characterization methods, device stability and reproducibility, FTPS, XPS, Photoluminescence, Kelvin probe measurement, NanoSIMS, $\mathrm{UV}$-vis spectra on full series of $\mathrm{Al}^{3+}$-doped perovskite.

\section{REFERENCES}

1 M. M. Lee, J. Teuscher, T. Miyasaka, T. N. Murakami and H. J. Snaith, Science (80-. )., 2012, 338, 643-647.

2 M. Liu, M. B. Johnston and H. J. Snaith, Nature, 2013, 501, 395-8.

3 J. H. Heo, S. H. Im, J. H. Noh, T. N. Mandal, C.-S. Lim, J. A. Chang, Y. H. Lee, H. Kim, A. Sarkar, M. K. Nazeeruddin, M. Grätzel and S. Il Seok, Nat. Photonics, 2013, 7, 486491.

4 N. J. Jeon, J. H. Noh, Y. C. Kim, W. S. Yang, S. Ryu and S. Il Seok, Nat. Mater., 2014, $13,1-7$.

5 M. Saliba, T. Matsui, J.-Y. Seo, K. Domanski, J.-P. Correa-Baena, M. K. Nazeeruddin, S. M. Zakeeruddin, W. Tress, A. Abate, A. Hagfeldt and M. Grä Tzel, Energy Environ. Sci., $2016,9,-$. 
6 J. M. Ball, M. M. Lee, A. Hey and H. J. Snaith, Energy Environ. Sci., 2013, 6, 1739.

7 W.-J. Yin, T. Shi and Y. Yan, Appl. Phys. Lett., 2014, 104, 063903.

8 M. L. Agiorgousis, Y.-Y. Sun, H. Zeng and S. Zhang, J. Am. Chem. Soc., 2014, 136, $14570-5$.

9 D. W. deQuilettes, S. M. Vorpahl, S. D. Stranks, H. Nagaoka, G. E. Eperon, M. E. Ziffer, H. J. Snaith and D. S. Ginger, Science (80-. )., 2015, 348, 683-6.

10 N. K. Noel, A. Abate, S. D. Stranks, E. Parrott, V. Burlakov, A. Goriely and H. J. Snaith, ACS Nano, 2014, 8, 140829182541001.

11 W. Zhang, S. Pathak, N. Sakai, T. Stergiopoulos, P. K. Nayak, N. K. Noel, A. A. Haghighirad, V. M. Burlakov, D. W. deQuilettes, A. Sadhanala, W. Li, L. Wang, D. S. Ginger, R. H. Friend and H. J. Snaith, Nat. Commun., 2015, 6, 10030.

12 J. Xu, A. Buin, A. H. Ip, W. Li, O. Voznyy, R. Comin, M. Yuan, S. Jeon, Z. Ning, J. J. McDowell, P. Kanjanaboos, J.-P. Sun, X. Lan, L. N. Quan, D. H. Kim, I. G. Hill, P. Maksymovych and E. H. Sargent, Nat. Commun., 2015, 6, 7081.

13 Y. Shao, Z. Xiao, C. Bi, Y. Yuan and J. Huang, Nat. Commun., 2014, 5, 5784.

14 W. Zhang, M. Saliba, D. T. Moore, S. K. Pathak, M. T. Hörantner, T. Stergiopoulos, S. D. Stranks, G. E. Eperon, J. A. Alexander-Webber, A. Abate, A. Sadhanala, S. Yao, Y. Chen, R. H. Friend, L. A. Estroff, U. Wiesner and H. J. Snaith, Nat. Commun., 2015, 6, 6142.

15 G. Kieslich, S. Sun and A. K. Cheetham, Chem. Sci., 2014, 5, 4712-4715.

16 I. Robinson and R. Harder, Nat. Mater., 2009, 8, 291-8.

17 A. Pramanick, X. P. Wang, C. Hoffmann, S. O. Diallo, M. R. V. Jørgensen and X.-L. 
Wang, Phys. Rev. B, 2015, 92, 174103.

G. . Williamson and W. . Hall, Acta Metall., 1953, 1, 22-31.

19 Y. Zhao and J. Zhang, J. Appl. Crystallogr., 2008, 41, 1095-1108.

20 D. T. Moore, H. Sai, K. W. Tan, D.-M. Smilgies, W. Zhang, H. J. Snaith, U. Wiesner and L. A. Estroff, J. Am. Chem. Soc., 2015, 137, 2350-8.

21 G. Kieslich, S. Sun and A. K. Cheetham, Chem. Sci., 2015, 6, 3430-3433.

22 R. A. Meyers, Ed., Encyclopedia of Analytical Chemistry, John Wiley \& Sons, Ltd, Chichester, UK, 2006.

23 R. A. Berner, Geochim. Cosmochim. Acta, 1975, 39, 489-504.

24 N. Kubota and J. W. Mullin, J. Cryst. Growth, 1995, 152, 203-208.

25 K. Sangwal, Prog. Cryst. Growth Charact. Mater., 1998, 36, 163-248.

26 J. J. De Yoreo, P. U. P. A. Gilbert, N. A. J. M. Sommerdijk, R. L. Penn, S. Whitelam, D. Joester, H. Zhang, J. D. Rimer, A. Navrotsky, J. F. Banfield, A. F. Wallace, F. M. Michel, F. C. Meldrum, H. Colfen and P. M. Dove, Science (80-. )., 2015, 349, aaa6760-aaa6760.

27 F. Deschler, M. Price, S. Pathak, L. E. Klintberg, D.-D. Jarausch, R. Higler, S. Hüttner, T. Leijtens, S. D. Stranks, H. J. Snaith, M. Atatüre, R. T. Phillips and R. H. Friend, J. Phys. Chem. Lett., 2014, 5, 1421-6.

28 S. D. Stranks, V. M. Burlakov, T. Leijtens, J. M. Ball, A. Goriely and H. J. Snaith, Phys. Rev. Appl., 2014, 2, 034007.

29 M. B. Johnston and L. M. Herz, Acc. Chem. Res., 2015, 49, 146-154. 
30 S. De Wolf, J. Holovsky, S.-J. Moon, P. Löper, B. Niesen, M. Ledinsky, F.-J. Haug, J.-H. Yum and C. Ballif, J. Phys. Chem. Lett., 2014, 5, 1035-1039.

31 P. Docampo, J. M. Ball, M. Darwich, G. E. Eperon and H. J. Snaith, Nat. Commun., 2013, 4, 2761 .

32 C.-C. Chueh, C.-Z. Li and A. K.-Y. Jen, Energy Environ. Sci., 2015, 8, 1160-1189.

33 S. van Reenen, M. Kemerink and H. J. Snaith, J. Phys. Chem. Lett., 2015, 6, 3808-3814.

34 H. J. Snaith, A. Abate, J. M. Ball, G. E. Eperon, T. Leijtens, N. K. Noel, S. D. Stranks, J. T.-W. W. Wang, K. Wojciechowski and W. Zhang, J. Phys. Chem. Lett., 2014, 5, 15111515.

35 Q. Wang, C. Bi and J. Huang, Nano Energy, 2015, 15, 275-280. 